relapse in patients with G3 HCV. Monocytes may act as a sanctuary site for HCV virions during interferon-based treatment, facilitating relapse after withdrawal of therapy.

Competing interests M Cunningham: None declared, A Javaid: None declared, J Waters: None declared, G Foster Grant/Research Support from: Roche, Janssen, Tibotec, Novartis, Consultant for: Abbott, BI, BMS, Chughai, Janssen, Merck, Novartis, Roche, Tibotec.

\section{PM0-168 EFFECTS OF LIDOCAINE 3\% GEL DELIVERED RECTALLY IN ANORECTAL DYSFUNCTION (ARD) INDUCED BY TELAPRAVIR THERAPY IN CHRONIC HEPATITIS C (CHC-C) A RANDOMISED PLACEBO CONTROL STUDY}

doi:10.1136/gutjnl-2012-302514b.168

\begin{abstract}
${ }^{1,2} \mathrm{P}$ Basu, ${ }^{*}{ }^{3} \mathrm{~T}$ Nair, ${ }^{3} \mathrm{~S}$ Farhat, ${ }^{4} \mathrm{M}$ Jafri, ${ }^{3} \mathrm{~K}$ Mittimani, ${ }^{3} \mathrm{~N}$ James Shah, ${ }^{3} \mathrm{~L}$ Ang, ${ }^{3} \mathrm{~S}$ foustin, ${ }^{3} \mathrm{~A}$ Anwarullah. ${ }^{1}$ Department of Gastroenterology and Hepatology, Columbia University, College of Physicians and Surgeons, New York, New York, USA; ${ }^{2}$ Gastroenterology, North Shore University, New York, USA; ${ }^{3}$ Internal Medicine, North Shore University, New York, USA; ${ }^{4}$ Internal Medicine, NYMC Richmond, New York, USA
\end{abstract}

Introduction Telapravir is a Potent Protease Inhibitor, which causes anorectal dysfunction (ARD) comprising Proctalgia, Rectal Ulcers, Hemorrhoids and rectal bleeding. Conventional therapy is suboptimal causing treatment Failure. This study evaluates 3\% Topical Lidocaine gel rectal delivery to abate the drug related ARD to avoid treatment failure.

Methods 52 Patients (mean age 51) were recruited undergoing therapy with Telapravir, Peg Interferon and Ribaviran for CHC-C. 45/52 (86\%), with Rectalgia, 8/52 (15\%) rectal ulcers, Hemorrhoids 19/52 (36\%) with bleeding 6/19 (31\%) without Bleeding 13/19 (68\%). Group A $(n=17)$ placebo Group B $(n=17)$ hydrocortisone 2.5\% Cream and Group C $(n=18)$ Lidocaine 3\% Gel foam per rectally twice daily. All underwent Pre and post Proctoscopic evaluation and Ano-rectal manometry.

Results

\begin{tabular}{lcrr}
\hline & Group A & \multicolumn{1}{c}{ Group B } & \multicolumn{1}{c}{ Group C } \\
\hline Rectalgia & $3 / 17(17 \%)$ & $8 / 17(47 \%)$ & $17 / 18(94 \%)$ \\
Rectal ulcers & $0 / 2(0 \%)$ & $1 / 3(33 \%)$ & $2 / 3(66 \%)$ \\
Hemorrhoids resolved w/o bleed & $1 / 6(16 \%)$ & $2 / 6(33 \%)$ & $5 / 7(71 \%)$ \\
Proctocopic examination showing normal- & $4 / 17(23 \%)$ & $7 / 17(41 \%)$ & $17 / 18(94 \%)$ \\
isation of mucosa post therapy & & &
\end{tabular}

Conclusion Results: Rectalgia resolved in 3/17 (17\%), 8/17 (47\%) and $7 / 18(94 \%)$ for Group A, B and C respectively. Rectal ulcers healed in $0 / 2(0 \%), 1 / 3(33 \%)$ and $2 / 3(66 \%)$ for all the above groups. Hemorrhoids resolved in 1/6 (16\%), 2/6 (33\%) and 5/7 (71\%) in all groups. Pre/Post Proctoscopy revealed normal mucosal integrity $4 / 17$ (23\%), 7/17 (41\%) and 17/18 (94\%) above groups. Results of Pre/ Post $\mathrm{Rx}$ mean scores for pain, Itching and Burning shown on (Abstract PMO-168 table 3). AR Manometry results showed Pre/ Post treatment high sphincter tone $>4 \mathrm{~mm}$ in Group A 2/15 (8\%) and no differences in pre and post treatment, Group B 7/15 (41\%), 4/15 (22\%) and Group C 5/15 (20\%), 2/15 (10\%) respectively (Abstract PMO-168 table 4). Side events; Numbness, 4/17 (23\%) in lidocaine. Conclusion: Rectally delivered Lidocaine 3\% gel is efficacious, tolerable compared to the SOC and placebo for ARD causing treatment failure, retention and SVR. Larger trial needs to validate this finding.

Competing interests None declared.
PM0-169 PEGYLATED INTERFERON $\alpha$, NITAZOXANIDE TELAPRAVIR, RIBAVIRIN, IN GENOTYPE 1 UNDERGOING PRIOR EXPERIENCED CHRONIC HEPATITIS C PATIENTS: A RANDOMISED PLACEBO CONTROL CLINICAL PILOT TRIAL (I N T R I G U E C) INTERIM

doi:10.1136/gutjnl-2012-302514b.169

${ }^{1,2} \mathrm{P}$ Basu, ${ }^{*}{ }^{3} \mathrm{~T}$ Nair, ${ }^{3} \mathrm{~S}$ Farhat, ${ }^{4} \mathrm{M}$ Jafri, ${ }^{3} \mathrm{~K}$ Mittimani, ${ }^{3} \mathrm{~N}$ James Shah, ${ }^{3} \mathrm{~L}$ Ang, ${ }^{3} \mathrm{~S}$ foustin. ${ }^{1}$ Department of Gastroenterology and Hepatology, Columbia University, College of Physicians and Surgeons, New York, New York, USA; ${ }^{2}$ Gastroenterology, North Shore University, New York, USA; ${ }^{3}$ Internal Medicine, North Shore University, New York, USA; ${ }^{4}$ Internal Medicine, NYMC Richmond, New York, USA

Introduction Chronic hepatitis $\mathrm{C}$ is a global challenge with end stage liver disease and rising Hepatocellular Carcinoma. Peg Interferon $\alpha$ and Ribavirin was the backbone of therapy. Recently introduced Directly Acting Antivirals -protease inhibitor has a promising role in escalating Sustained Viral Response in Response guided therapy in non-responders, partial and relapses. This study utilised Nitazoxanide (NTZ) and Telapravir, with SOC for 24 weeks in treatment experienced patients.

Methods $50(\mathrm{n}=50)$ patients were divided into Group A $(\mathrm{n}=12)$ NTZ 500 mg three times for 12 weeks, Group B $(n=12)$ NTZ 500 mg twice daily for 24 weeks Group $C(n=26)$ control. All received Peg Interferon $\alpha 2 \mathrm{a} 180 \mu \mathrm{g}$ SQ QOW with fixed dose of Ribavirin $1200 \mathrm{mg}$ daily for 24 weeks with Telapravir $750 \mathrm{mg}$ three times daily for 12 weeks. Viral load was obtained at day 0,7 th day, 14 th day, 4 weeks, 12th week and 24 weeks. Viral kinetics was analysed. In Group A: 5/12 (42\%) Non-Responder, 6/12 (50\%) partial responder, 2/12 (16\%) relapsers. In Group B: 5/12 (42\%) Nonresponders, $6 / 12(50 \%)$ partial responder, $1 / 12$ relapsers $(8 \%)$. In Group C: $10 / 26$ (38\%) non-responder, 10/26 (38\%) partial responder, 4/26 (15\%) relapsers, 2/26 (8\%) unknown. Exclusion: Decompensated Cirrhotic, HCC, poor DM, Haemolytic Anaemia, Severe Coronary artery disease, major depression, renal failure, Prior severe skin rash, active drug and alcohol abuse. Side Effects: Anaemia 28/50 (56\%), Neutropenia 14/50 (28\%), Thrombocytopaenia 8/50 (16\%), Fatigue $34 / 50$ (68\%), Depression 10/50 (20\%), Mild skin rash 22/50 (44\%), Severe skin rash $1 / 50(2 \%)$. Use of Growth factors: Epogen $12 / 50$ (24\%) Neupogen 8/50 (16\%) Elthrombopag 5/50 (10\%).

\section{Results}

\begin{tabular}{lccr}
\hline Results & Group A & Group B & \multicolumn{1}{c}{ Group C } \\
\hline Undetectable & $9 / 12(75 \%)$ & $10 / 12(83 \%)$ & $16 / 26(62 \%)$ \\
NR & $1 / 12(8 \%)$ & $2 / 12(16 \%)$ & $4 / 26(15 \%)$ \\
PR & $1 / 12(8 \%)$ & $12 / 12(100 \%)$ & $3 / 26(11 \%)$ \\
AVR & $11 / 12(92 \%)$ & $12 / 12(100 \%)$ & $20 / 26(77 \%)$ \\
VRVR & $11 / 12(92 \%)$ & $10 / 12(83 \%)$ & $22 / 26(84 \%)$ \\
RVR & $9 / 12(75 \%)$ & $10 / 12(83 \%)$ & $18 / 26(70 \%)$ \\
EVR & $9 / 12(75 \%)$ & $10 / 12(83 \%)$ & $16 / 26(62 \%)$ \\
ETVR & $9 / 12(75 \%)$ & $10 / 12(83 \%)$ & $16 / 26(62 \%)$ \\
\hline
\end{tabular}

Conclusion This quadruple truncated regimen has excelled the RVR, ETVR over SOC with Directly Acting Antivirals over 13\%, without any difference between 24 weeks of NTZ over 12 . Needs a larger trial for validation.

Competing interests None declared.

PMO-170 PREVALENCE OF HEPATITIS E IN NEW YORK AMONG HIV
NEGATIVE CHRONIC LIVER DISEASE POPULATION “IS IT
AN INNOCENT BYSTANDER"
doi:10.1136/gutjnl-2012-302514b.170

${ }^{1,2} \mathrm{P}$ Basu, ${ }^{*}{ }^{3} \mathrm{~T}$ Nair, ${ }^{3} \mathrm{~S}$ Farhat, ${ }^{4} \mathrm{M}$ Jafri, ${ }^{3} \mathrm{~K}$ Mittimani, ${ }^{3} \mathrm{~N}$ James Shah, ${ }^{3} \mathrm{~L}$ Ang. ${ }^{1}$ Department of Gastroenterology and Hepatology, Columbia University, College of 
Physicians and Surgeons, New York, New York, USA; ${ }^{2}$ Gastroenterology, North Shore University, New York, USA; ${ }^{3}$ Internal Medicine, North Shore University, New York, USA; ${ }^{4}$ Internal Medicine, NYMC Richmond, New York, USA

Introduction Hepatitis $\mathrm{E}$ is essentially an oral fecal infection with high prevalence in developing countries. There is limited data available on its prevalence in Urban US. The study evaluates the prevalence of hepatitis E antibody IgG and IgM in New York in patients with chronic liver disease.

Methods $440(n=440)$ were divided into two groups: group $A$; control 140 patients without any stigmata of liver disease. Group B, 300 patients with history of liver disease including hepatitis B 125/ 300 (41\%), Chronic hepatitis C 60/300 (20\%), fatty liver 70/300 (23\%), Alcoholic liver disease 29/300 (10\%), HBV HCV co infection $9 / 300$ (3\%), auto immune hepatitis $2 / 300$ (0.6\%), PBC /PSC 5/300 $(2 \%)$. HEV antibody IGM and IGG were measured with HEV genotyping. 22/300 (7\%) patients were liver transplant recipients, 6/ $300(2 \%)$ patients were kidney transplant recipients. 43/300 (14\%) patients were Intra venous Drug Abuser. 20/300 (7\%) received Blood products.

Results History and genotypic characteristics

\begin{tabular}{lllll}
\hline Characteristics & $\begin{array}{l}\text { Chronic } \\
\text { hepatitis C }\end{array}$ & Chronic hepatitis B & Fatty liver & $\begin{array}{l}\text { Alcoholic liver } \\
\text { disease }\end{array}$ \\
\hline IVDU & $36 / 43(83 \%)$ & $7 / 43(16 \%)$ & NA & NA \\
Promiscuity & $23 / 42(52 \%)$ & $10 / 42(23 \%)$ & NA & $9 / 42(2 ! \%)$ \\
Transfusion & $6 / 20(30 \%)$ & $14 / 20(70 \%)$ & NA & NA \\
Genotype & & & & \\
G1 & $2 / 30(7 \%)$ & $60 / 69(87 \%)$ & $3 / 10(30 \%)$ & $1 / 13(7 \%)$ \\
G2 & $1 / 30(3 \%)$ & $8 / 69(11 \%)$ & $3 / 10(30 \%)$ & $4 / 13(30 \%)$ \\
G3 & $26 / 30(87 \%)$ & $1 / 69(1 \%)$ & $4 / 10(40 \%)$ & $8 / 13(62 \%)$ \\
G4 & $1 / 30(3 \%)$ & NA & NA & NA \\
G4 & NA & NA & NA & NA \\
\hline
\end{tabular}

Conclusion Results: Group (A) HEV IgG positive 13\% (18/140), Group B HEV IgG positive 40.6\% (122/300) including 3.7\% (4/122) having both IgM and IgG positive. The prevalence of HEV IgG was $54 \%(12 / 22)$ in the liver transplant recipient group and 33\% (2/6\%) kidney transplant recipient group. The study demonstrates the prevalence of hepatitis E infection in New York and HEV antibody in CLD. Including transplant donors and recipients. Question remains the impact and progression of acute or chronic liver disease with concomitant HEV in pre, peri, and post liver transplant recipient. Larger study needs to validate.

Competing interests None declared

\section{PM0-171 A PILOT OF TARGETED CASE FINDING FOR HEPATITIS B VIRUS (HBV) AND HEPATITIS C (HCV) IN THE BRITISH SOUTH ASIAN COMMUNITY}

doi:10.1136/gutjnl-2012-302514b.171

\footnotetext{
${ }^{1,2} \mathrm{~S}$ Mcpherson, ${ }^{*}{ }^{3} \mathrm{~S}$ Moses, ${ }^{3} \mathrm{M}$ Valappil, ${ }^{4} \mathrm{~S}$ Rehman, ${ }^{1} \mathrm{C}$ Miller, ${ }^{1} \mathrm{~K}$ Baxter, ${ }^{5} \mathrm{~A}$ Saeed, ${ }^{6} \mathrm{~K}$ Shafiq, ${ }^{1,2} \mathrm{M}$ Hudson, ${ }^{1,2} \mathrm{M}$ Bassendine. 'Liver Unit, Freeman Hospital, Newcastle upon Tyne, UK; ${ }^{2}$ Institute of Cellular Medicine, Newcastle University, Newcastle upon Tyne, UK; ${ }^{3} H P A$, Newcastle upon Tyne, UK; ${ }^{4}$ Broadway Medical Centre, Newcastle upon Tyne, UK; ${ }^{5}$ Queen Elizabeth Hospital, Gateshead, UK; ${ }^{6}$ Sunderland Royal Hospital, Sunderland, UK
}

Introduction Chronic HBV (cHBV) and HCV (cHCV) are frequent causes of cirrhosis and liver cancer. $\mathrm{cHBV}$ and $\mathrm{cHCV}$ are prevalent in subjects from South Asian countries and many infected individuals are unaware of their condition. A previous case finding pilot for $\mathrm{HBV}$ in the British-Chinese community of the NE of England found that $9 \%$ of subjects tested were $\mathrm{HBsAg}$ positive. ${ }^{1}$ Targeted screening for
HBV and HCV is recommended by the AASLD ${ }^{2}$ and the European Liver Patients Association ${ }^{3}$ in subjects born in endemic areas (HBsAg prevalence $\geq 2 \%$ ). However, there are no current UK guidelines. Our aim was to apply AASLD recommendations to British South Asian community of NE of England.

Methods Members of the NE South Asian community were invited to attend screening sessions at a Newcastle Mosque and the Sunderland Bangladeshi Community Centre. Dry blood spots were obtained by finger prick and tested for $\mathrm{HBsAg}, \mathrm{HBcAb}$ (Abbott ARCHITECT ${ }^{\circledR}$ ) and HCV antibody (Roche + confirmed by Biorad). $\mathrm{HBsAg}$ and/or HCV Ab positive individuals were advised to undergo confirmatory testing and be referred for specialist assessment.

Results To date, 329 subjects were screened in four sessions (median age 39 , range $6-83 ; 75 \%$ male). The screened subjects were most frequently born in Bangladesh (49\%) followed by Pakistan $(36 \%)$, the UK $(14 \%)$ or other countries $(6 \%) .1 .8 \%$ of subjects were $\mathrm{HBsAg}$ positive indicating $\mathrm{cHBV} .2 .8 \%$ of subjects had past infection with $\mathrm{HBV}$ ( $\mathrm{HBs} \mathrm{Ag}$ negative, $\mathrm{HBcAb}$ positive). $1 \%$ of subjects were $\mathrm{HCV}$ $\mathrm{Ab}$ positive (all born in Pakistan). The prevalence of $\mathrm{cHBV}$ was 3\% for individuals born in Pakistan and $1.5 \%$ for those born in Bangladesh. 3\% of individuals born in Pakistan were HCV Ab positive. Only $4 \%$ of subjects reported previous vaccination against HBV.

Conclusion Undiagnosed cHBV is prevalent in the South Asian community of NE England, although the prevalence was lower than in the British-Chinese community. These results provide evidence for a targeted case finding programme for HBV and HCV in subjects born in South Asia.

Funding This study was supported by a Gilead UK and Ireland Fellowship and Livernorth.

Competing interests None declared.

\section{REFERENCES}

1. McPherson S, et al. Gut 2011;60(Supp 2):A25

2. Lok A, McMahon B. Hepatology 2009;50:1-36.

3. Piorkowsky N. J Hepatol 2009;51:1068-73.

\section{PM0-172 DIRECT NON-INVASIVE SERUM MARKERS OF LIVER FIBROSIS PREDICT FIBROSIS EVOLUTION IN CHRONIC HEPATITIS C BUT ARE INCREASED BY INTERFERON-BASED THERAPY}

doi:10.1136/gutjnl-2012-302514b.172

${ }^{1} \mathrm{~S}$ Tanwar, ${ }^{*}{ }^{1} \mathrm{P} M$ Trembling, ${ }^{2} \mathrm{E}$ Ellis, ${ }^{2} \mathrm{~J}$ Parkes, ${ }^{3} \mathrm{C}$ Herold, ${ }^{4} \mathrm{D}$ Schuppan, ${ }^{1}$ W M C Rosenberg. ${ }^{1}$ Centre for Hepatology, University College London (UCL), London, UK; ${ }^{2}$ Public Health Sciences and Medical Statistics, University of Southampton, Southampton, UK; ${ }^{3}$ University of Erlangen, Erlangen, Germany; ${ }^{4}$ Department of Internal Medicine, University of Mainz, Mainz, Germany

Introduction Liver biopsy remains the reference standard for the detection of liver fibrosis. While non-invasive markers of liver fibrosis have been validated in chronic hepatitis $\mathrm{C}$, their performance during interferon-based therapy is not established.

Methods 70 previous non-responders to interferon based therapy for chronic hepatitis C (40 male, age range $24-67$, mean 48.7) were recruited from five centres. Patients were randomised to receive pegylated interferon with or without silymarin for 24 months as an exploratory antifibrotic therapy. All patients underwent a liver biopsy and ELF tests (HA, P3NP, TIMP-1) prior to, and after treatment (month 0 and 24). Changes in histological fibrosis stages before and after therapy ( 0 to 24 months) were compared with changes in marker scores before, during and after therapy (0 to 24 months).

Results Mean ELF score prior to therapy was 9.32 (SD 1.10), during therapy 9.91 (SD1.18) and after therapy 9.53 (SD 1.27). ELF scores 